\title{
Static Theory of the Giant Quadrupole Resonance in Deformed Nuclei*
}

\author{
Michael Danos \\ National Bureau of Standards, Washington, D. C. \\ WALTER GREINER \\ Institut für Theoretische Physik, University of Frankfurt, Frankfurt/Main, Germany \\ AND \\ C. BYRON KOHR $\dagger$ \\ University of Maryland, College Park, Maryland \\ (Received 17 May 1966)
}

\begin{abstract}
The modes and frequencies of the giant quadrupole resonance of heavy deformed nuclei have been calculated. The quadrupole operator is computed and the absorption cross section is derived. The quadrupole sum rule is discussed, and the relevant oscillator strengths have been evaluated for various orientations of the nucleus. The giant quadrupole resonances have energies between 20 and $25 \mathrm{MeV}$. The total absorption cross section is about $20 \%$ of the giant dipole absorption cross section. Of particular interest is the occurrence of the quadrupole mode which is sensitive to the nuclear radius in a direction of approximately $\theta=\frac{1}{4} \pi$ from the symmetry axis. This may give information on the details of the nuclear shape.
\end{abstract}

\section{INTRODUCTION}

$T$ HE predictions of the hydrodynamic model of the giant dipole resonance have been found to agree with the experimental facts in very great detail. ${ }^{1-6}$ As a matter of fact, no real discrepancies between the predictions of the model and experimental results have as yet turned up. It thus seems important to develop the model further in order to find its limits of validity.

In this paper we develop the theory of the giant quadrupole resonance for deformed nuclei with a rigid boundary condition, the so-called "static" theory. This step is analogous to the static theory of the splitting of the giant dipole resonance. ${ }^{7,8} \mathrm{We}$ shall see that the splitting of the quadrupole resonance is of the order of 5-6 MeV compared with about half that amount for the giant dipole resonance. The splitting of the giant quadrupole resonance is of interest also in the following connection. In the same way as the giant dipole resonance is sensitive to the nuclear axes in three orthogonal directions, the giant quadrupole resonance is sensitive to the nuclear radius in five directions. Thus,

\footnotetext{
* Work supported in part by the German Bundesministerium fur Wissenschaftliche Forschung and the U. S. Office of Naval Research.

† Present address: Millersville State College, Millersville, Pennsylvania. Work done in partial fulfillment of the requirements for the Doctor of Philosophy degree in physics at the University of Maryland.

${ }^{1}$ M. Danos and W. Greiner, Phys. Rev. 134, B284 (1964).

2 M. Danos, W. Greiner, and C. B. Kohr, Phys. Rev. 138, B1055 (1965).

${ }^{3}$ E. Ambler, E. G. Fuller, and H. Marshak, Phys. Rev. 138, B117 (1965).

${ }^{4} \mathrm{~J}$. LeTourneux, Kgl. Danske Videnskab. Selskab, Mat. Fys. Medd. 34, No. 11 (1965).

${ }^{5}$ D. S. Fielder, J. LeTourneux, K. Min, and W. D. Whitehead, Phys. Rev. Letters 15, 33 (1965).

${ }^{6}$ M. G. Huber, H. J. Weber, M. Danos, and W. Greiner, Phys. Rev. Letters 15, 529 (1965).

${ }^{7}$ K. Okamoto, Phys. Rev. 110, 143 (1958).

8 M. Danos, Nucl. Phys. 5, 23 (1958).
}

if the model is still applicable, the giant quadrupole resonance, together with the giant dipole resonance, can reveal rather fine details of the nuclear shape.

In this paper we do not treat the rotations and vibrations of the nucleus. The results obtained are thus valid only for the coordinate system fixed relative to the nucleus, the so-called intrinsic coordinate system. They would be valid also for the laboratory system for a nucleus with infinite spin which could have a fixed direction in the laboratory. The obtained eigenenergies have the same meaning and usefulness as those of the equivalent step in the theory of the giant dipole resonance. They give the qualitative picture, and they serve as input parameters to the complete theory which treats the giant resonances together with the vibrations and rotations.

The experimental situation concerning the giant quadrupole resonance is still almost void of results; this is so mostly because of the experimental difficulties (the giant quadrupole resonance lies on the back of the much larger giant dipole resonance). It is, however, of great interest to determine whether or not the hydrodynamic model is still applicable for the giant quadrupole resonance, i.e., whether or not this resonance actually exists with a width comparable to that of the giant dipole resonance ${ }^{9}$ as assumed in a previous communication. ${ }^{10}$ This would have to be expected if the hydrodynamic model is still applicable at the energy of the giant quadrupole resonance which is about 1.6 times higher than the giant dipole resonance. The breakdown of the hydrodynamic model should be expected at an energy where the wavelength of the "second sound" (the oscillation in the relative velocity between protons and neutrons) approaches the order of the characteristic length of the short range two-body

\footnotetext{
${ }^{9} \mathrm{M}$. Danos and W. Greiner, Phys. Rev. 138, B876 (1965).

${ }_{10}$ M. Danos, W. Greiner, and C. B. Kohr, Phys. Letters 12.344 (1964).
} 


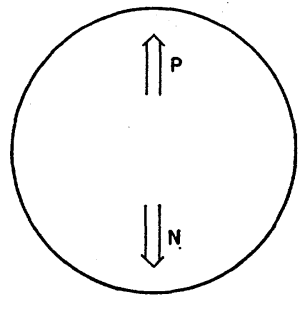

DIPOLE

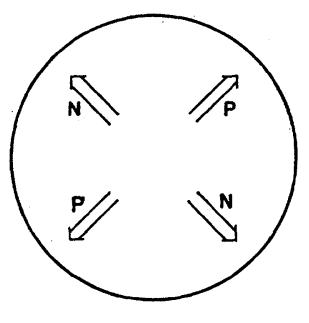

QUADRUPOLE
FIG. 1. Schematic showing the motion of protons and neutrons for the giant dipole and giant quadrupole vibrations in a spherical nucleus.

correlations of the nuclear wave functions ${ }^{11}$ which is about $10^{-13} \mathrm{~cm}$. The "second-sound" wavelength of the giant dipole resonance is about $\chi=k^{-1} \approx 2.7 \times 10^{-13} \mathrm{~cm}$, while it is only about $X \approx 1.7 \times 10^{-13} \mathrm{~cm}$ for the giant quadrupole resonance, i.e., it is already quite close to the two-body correlation length. It must be emphasized at this point that this breakdown is not limited to the hydrodynamic model. For identically the same reasons, the long-range correlation shell-model description, nowadays called the "particle-hole" picture, becomes inadequate at higher energies (or larger momentum transfers in electron scattering). There the quasideuteron effect becomes the predominant mechanism. We hope by this discussion to stimulate some interest among our experimental friends regarding this energy region.

Briefly, the paper is organized as follows. In Sec. II the general results of the hydrodynamic model are given. In Sec. III three methods for obtaining the solution to the Helmholtz equation in a deformed nucleus are discussed. In this section we also evaluate the quadrupole energies for a spherical nucleus. In Sec. IV the expression of the photon quadrupole operator in terms of the collective coordinates is derived, and in Sec. $\mathrm{V}$ the photon absorption cross section is calculated and the sum rule is established. Section VI exhibits the computation of oscillator strengths in the intrinsic system for various cases. Finally, we have summarized the results and discussed their physical aspects in Sec. VII.

\section{THE HYDRODYNAMICAL MODEL}

In the hydrodynamic model ${ }^{12,13}$ it is assumed that the internal motion consists of a flow of protons and neutrons in opposite directions such that the changes in the proton and neutron densities, $\rho_{p}$ and $\rho_{n}$, cancel in the total nuclear density, $\rho_{0}$. The static theory in addition considers the nuclear surface to remain fixed. The restoring force is supplied by the symmetry energy.

${ }^{11}$ J. H. D. Jensen, Angew. Chemie 76, 69 (1964).

12 J. H. D. Jensen and H. Steinwedel, Z. Naturforsch. 5a, 413 (1950).

${ }_{13}$ M. Danos, Ann. Physik 6, 265 (1952).
Writing

$$
\rho_{p}=\rho_{p}(0)\left[1+\eta(\mathbf{r}) e^{-i \omega t}\right], \quad \rho_{n}=\rho_{0}-\rho_{p},
$$

the motion is described by

$$
\Delta \eta+k^{2} \eta=0
$$

together with the boundary condition appropriate to a rigid surface,

$$
\begin{aligned}
& \text { Here } \\
& \qquad \begin{aligned}
\left.\hat{n} \cdot \nabla \eta\right|_{r=R}=\left.\hat{n} \cdot \mathfrak{f}\right|_{r=R} . \\
k^{2}=\omega^{2} u^{-2}(1+i \Gamma / \omega), \\
\mathfrak{f}=e\left(\varepsilon \rho_{0} / 8 \kappa\right) \rho_{p}(0), \\
u^{2}=8 \kappa N Z / M^{*} A^{2}, \\
M^{*}=M /(1+\alpha) .
\end{aligned}
\end{aligned}
$$

In these expressions $\kappa$ is the symmetry energy constant, $M^{*}$ is the effective nucleon mass introduced to allow for virtual meson exchange, ${ }^{11,14} \mathcal{E}$ is the externally applied electric field strength, and $\Gamma$ is the width of the resonance. In Eq. (3) $n$ is a unit vector normal to the surface.

The dipole and quadrupole modes are schematically depicted in Fig. 1.

\section{THE SOLUTION TO THE HELMHOLTZ EQUATION}

Owing to the $\gamma$ vibrations of the nuclear surface, the instantaneous shape of the nucleus is in general triaxial. Therefore, no tabulated functions are available to express the solutions of (2) in closed form. We shall use two approximation methods to obtain solutions and check the results in the limiting case of axial symmetry against the exact solutions in an ellipsoidal nucleus where tabulated spheroidal wave functions are available. In the first of the two approximation methods which we refer to as the expansion method, we treat the wave equation in spherical coordinates. The general solution is then expressed as a linear superposition of spherical waves. The expansion is performed to first order in the nuclear deformation parameters. The eigenvalues are obtained upon satisfying the boundary conditions. For the second method we use a variational technique to obtain the eigenvalues of the deformed state.

\section{A. The Expansion Method}

When the wave equation is expressed in spherical coordinates, the general solution that is finite at the origin is

$$
\eta(\mathbf{r})=\sum_{L=0}^{\infty} \sum_{M=-L}^{L} C_{L M} j_{L}(k r) Y_{L M}(\theta, \varphi)
$$

${ }^{14}$ M. Danos, University of Maryland Technical Report No. 221, 1961 (unpublished). 
The origin of the coordinate system is the center of mass of the nucleus. In this approach we calculate the gradient of $\eta(\mathbf{r})$ and equate the scalar product $\hat{n} \cdot \nabla \eta(\mathbf{r})$ and the scalar product $\hat{n} \cdot \mathfrak{f}$, both of which are evaluated at the surface. In this manner we obtain the coefficients $C_{L M}$ in terms of the electric field and the wave number $k$. By finding the poles of these coefficients as functions of the energy, we obtain the eigenvalues.

To calculate the $\nabla \eta(\mathbf{r})$ we use the gradient formula ${ }^{15}$ $\begin{aligned} \nabla j_{L}(k r) Y_{L M}= & k\left\{[(L+1) /(2 L+1)]^{1 / 2} j_{L+1}(k r) \mathbf{T}_{L, L+1, M}\right. \\ & \left.+[L /(2 L+1)]^{1 / 2} j_{L-1}(k r) \mathbf{T}_{L, L-1, M}\right\}, \quad(9)\end{aligned}$ where the vectorial spherical harmonics are defined by

$$
\mathbf{T}_{L \lambda M}=\sum_{\mu=-1}^{1}(1 \lambda L \mid-\mu, M+\mu, M) Y_{\lambda M+\mu} \xi_{-\mu} .
$$

The $\xi_{\mu}$ are the spherical basis vectors

$$
\xi_{ \pm 1}=\mp(1 / \sqrt{2})(\hat{x} \pm i \hat{y}), \quad \xi_{0}=\hat{z}
$$

with the orthonormality relations

$$
\xi_{\mu} \cdot \xi_{\nu}=(-1)^{\mu} \delta_{\mu-\nu}, \quad \xi_{\mu}^{*} \cdot \xi_{\nu}=\delta_{\mu \nu} .
$$

The gradient of $\eta(\mathbf{r})$ is calculated by applying Eq. (9) to Eq. (8). The obtained expressions, together with several other long formulas, are given in Appendix A.

We express the normal unit vector in terms of the spherical unit vectors as

$$
\hat{n}=\sum_{\mu=-1}^{1} \alpha_{\mu} \xi_{\mu}
$$

and consider the coefficients $\alpha_{\mu}$ as functions of the surface deformation parameters, $a_{0}$ and $a_{2}$, defined in the usual manner

$$
R=R_{0}\left[1+a_{0} Y_{20}+a_{2}\left(Y_{22}+Y_{2-2}\right)\right] .
$$

Expressions for the $\alpha_{\mu}$ are given in Appendix A.

We still need to evaluate the spherical Bessel functions at the surface, Eq. (14). We do this by expanding the spherical Bessel functions $j_{\lambda}(k R)$ in a Taylor series about $R=R_{0}$ and retain only terms to first order in the deformation parameters. We have

$$
\begin{aligned}
j_{\lambda}(k R) \approx j_{\lambda}\left(k R_{0}\right)+j_{\lambda}{ }^{\prime}( & \left(k R_{0}\right)\left(k R_{0}\right) \\
& \times\left[a_{0} Y_{20}+a_{2}\left(Y_{22}+Y_{2-2}\right)\right] .
\end{aligned}
$$

The prime denotes differentiation with respect to the argument $z=k R$. The expression for the normal gradient obtained in this way is again given in Appendix A.

We now evaluate $\hat{n} \cdot \mathfrak{f}$ at the surface. Let us consider a plane, circularly polarized wave traveling in the direction $\mathbf{s}$, with wave number $s=|\mathbf{s}|$. Then

and

$$
\mathcal{E}=\mathcal{E}_{0} \hat{u}_{p} e^{i \mathrm{~s} \cdot \mathrm{r}}
$$

$$
\hat{u}_{p}=(1 / \sqrt{2})\left(\hat{u}_{1}+i P \hat{u}_{2}\right),
$$

${ }^{15}$ M. E. Rose, Elementary Theory of Angular Momentum (John Wiley \& Sons, Inc., New York, 1957). with $\hat{u}_{1}, \hat{u}_{2}$, and $\mathrm{s} / \mathrm{s}$ real unit vectors forming a righthanded orthogonal Cartesian coordinate system. $P$ is the polarization index with $P=1$ corresponding to left circularly polarized waves, and $P=-1$ to right circularly polarized waves.

Using the well-known expansion for a plane wave

$$
e^{i s \cdot r}=\sum_{L} i^{L}[4 \pi(2 L+1)]^{1 / 2} j_{L}(s r) Y_{10}(\theta, \varphi),
$$

we obtain the multipole expansion of a circularly polarized plane wave $\mathrm{e}^{15}$ in the coordinate system used in Eq. (14), i.e., in the "intrinsic coordinate system,"

$$
\begin{aligned}
\boldsymbol{\varepsilon}=\mathcal{E}_{0} \pi \sum_{L} \sum_{M} i^{L}(2 L+1)^{1 / 2} & \mathscr{D}_{M P}{ }^{(L)}(\alpha \beta \gamma) \\
& \times\left(\mathbf{A}_{L M}{ }^{m}+i^{P} \mathbf{A}_{L M^{e}}\right),
\end{aligned}
$$

where

$$
\begin{aligned}
\mathbf{A}_{L M}{ }^{m}=- & j_{L}(s r) \mathbf{T}_{L L M}, \\
\mathbf{A}_{L M}{ }^{e}=- & {[L /(2 L+1)]^{1 / 2} j_{L+1} \mathbf{T}_{L, L+1, M} } \\
& +[(L+1) /(2 L+1)]^{1 / 2} j_{L-1} \mathbf{T}_{L, L-1, M} .
\end{aligned}
$$

The $\mathfrak{D}_{M P}{ }^{(L)}(\alpha \beta \gamma)$ are the rotation matrices and $\alpha, \beta$, and $\gamma$ are the Euler angles.

We wish to consider a plane polarized wave. We thus must consider an appropriate superposition of left circularly polarized and right circularly polarized waves. For $\alpha=\beta=\gamma=0$ we choose $\boldsymbol{\varepsilon}$ along the $x$ axis and $\mathbf{s}$ along the $z$ axis. Thus

$$
\begin{aligned}
& \mathcal{E}=\mathcal{E}_{0}(\pi / \sqrt{2}) \sum_{L} \sum_{M} i^{L}(2 L+1)^{1 / 2} \\
& \times\left[\mathbf{A}_{L M}{ }^{m}\left(D_{M 1}{ }^{(L)}+D_{M-1}{ }^{(L)}\right)\right. \\
& \left.+i \mathbf{A}_{L M}{ }^{e}\left(D_{M 1}{ }^{(L)}-D_{M-1}{ }^{(L)}\right)\right] .
\end{aligned}
$$

Finally, keeping only the quadrupole term we have

$$
\boldsymbol{E}=-\mathcal{E}_{0} \pi\left(\frac{5}{2}\right)^{1 / 2} \sum_{M=-2}^{2}\left(D_{M+}{ }^{(2)} \mathbf{A}_{2 M^{m}}+i D_{M-}{ }^{(2)} \mathbf{A}_{2 M^{e}}\right),
$$

where

$$
\mathscr{D}_{M \pm}{ }^{(2)}=\mathscr{D}_{M 1}{ }^{(2)} \pm \mathscr{D}_{M-1}{ }^{(2)} .
$$

For energies of the order of $25 \mathrm{MeV}$, we have $s R_{0}$ $=E R_{0} / \hbar c \approx 0.9$. Hence, we may neglect $j_{3}(s r)$ in our calculations since it will be at most only about $3 \%$ of $j_{1}(s r)$.

Using the expression for the vectorial spherical harmonics, and Eqs. (19), (20), and (21), we obtain

$$
\begin{aligned}
\mathcal{E}= & -\mathcal{E} \pi\left(\frac{5}{2}\right)^{1 / 2} \sum_{M=-2}^{2} \sum_{\mu=-1}^{1}\left[-D_{M+}{ }^{(2)} j_{2}(s r)\right. \\
& \times(122 \mid-\mu, M+\mu, M) Y_{2}{ }_{M+\mu}-i\left(\frac{3}{5}\right)^{1 / 2} \mathscr{D}_{M-}{ }^{(2)} j_{1}(s r) \\
& \left.\times(112 \mid-\mu, M+\mu, M) Y_{1 M+\mu}\right] \xi_{-\mu} .
\end{aligned}
$$

Let us now consider the scalar product $\hat{n} \cdot \boldsymbol{\varepsilon}$. Since $\hat{n}$ contains the angular functions $Y_{1 v}$ and $Y_{3 v}$ only, in combining with $Y_{2 \mu}$ the parity rule will restrict the scalar product to terms such as $Y_{1 \nu^{\prime}}, Y_{3 \nu^{\prime}}$, and $Y_{5 \nu^{\prime}}$, while in combining with $Y_{1 \mu}$, we will obtain only terms $Y_{00}, Y_{2 \nu^{\prime}}$, and $Y_{4{ }^{\prime}}$. Since we are interested only in the 
TABLE I. Directions of the incident photon and electric field, and the related quadrupole modes which are excited.

\begin{tabular}{cccccc}
\hline \hline$\alpha$ & $\beta$ & $\gamma$ & $\mathbf{s}$ & $\mathcal{E}$ & $\begin{array}{c}\text { Excited } \\
\text { modes } \\
\left(a_{0}=a_{2}=0\right)\end{array}$ \\
\hline 0 & 0 & 0 & $s \hat{z}$ & $\varepsilon_{0} \hat{x}$ & $\left(Y_{21}-Y_{2-1}\right)$ \\
$\frac{1}{2} \pi$ & 0 & 0 & $s \hat{z}$ & $\varepsilon_{0} \hat{y}$ & $\left(Y_{21}+Y_{2-1}\right)$ \\
0 & $\frac{1}{4} \pi$ & 0 & $\frac{1}{2} s \sqrt{2}(\hat{x}+\hat{z})$ & $\frac{1}{2} \varepsilon_{0} \sqrt{2}(\hat{x}-\hat{z})$ & $Y_{20}$ and \\
$\left(Y_{22}+Y_{2-2}\right)$ \\
$\frac{1}{4} \pi$ & $\frac{1}{4} \pi$ & 0 & $\frac{1}{2} s(x+\hat{y}+\sqrt{2} \hat{z})$ & $\left(\varepsilon_{0} / 2\right)(\hat{x}+\hat{y}-\sqrt{2} \hat{z})$ & $Y_{20}$ and \\
$\left(Y_{22}-Y_{2-2}\right)$ \\
\hline \hline
\end{tabular}

quadrupole oscillations, we need only the terms involving $Y_{1 \mu}$ in Eq. (25). The scalar product with the normal is then

$$
\begin{aligned}
\hat{n} \cdot \mathcal{E}= & -i \mathcal{E}_{0} \pi\left(\frac{2}{3}\right)^{1 / 2} j_{1}(s r)\left\{\left[-\alpha_{-1} \mathscr{D}_{0-}{ }^{(2)}(6)^{-1 / 2}\right.\right. \\
& \left.+\alpha_{0} \mathscr{D}_{-1-}{ }^{(2)}(2)^{-1 / 2}-\alpha_{+1} \mathfrak{D}_{-2-}{ }^{(2)}\right] Y_{1,-1} \\
& +\left[-\alpha_{-1} D_{1-}{ }^{(2)}(2)^{-1 / 2}+\alpha_{0} \mathscr{D}_{0-}{ }^{(2)}\left(\frac{2}{3}\right)^{1 / 2}\right. \\
& \left.-\alpha_{+1} D_{-1-}{ }^{(2)}\right] Y_{10}+\left[-\alpha_{-1} D_{2-}{ }^{(2)}\right. \\
& \left.\left.+\alpha_{0} D_{1-}{ }^{(2)}(2)^{-1 / 2}-\alpha_{+1} D_{0-}{ }^{(2)}(6)^{-1 / 2}\right] Y_{11}\right\} .
\end{aligned}
$$

To evaluate Eq. (26) at the surface, we again perform a Taylor expansion of $j_{1}(s R)$ to first order in $a_{\nu}$ about the point $s R_{0}$.

$$
\begin{aligned}
j_{1}(s R) \approx j_{1}\left(s R_{0}\right)+\left(s R_{0}\right) & j_{1}^{\prime}\left(s R_{0}\right) \\
\times & {\left[a_{0} Y_{20}+a_{22}\left(Y_{22}+Y_{2-2}\right)\right] . }
\end{aligned}
$$

The prime denotes differentiation with respect to the argument $\xi=s R$. We give the resulting expressions in Appendix A.

To evaluate the coefficients $C_{L M}$ for $L=2$, we consider the boundary condition Eq. (2). These coefficients may then be obtained by equating the corresponding coefficients of the $Y_{\lambda \mu}$. This procedure will produce a set of simultaneous equations. From these equations we obtain to first order in $a_{0}$ and $a_{2}$

with

$$
\begin{aligned}
& C_{20}=B_{20} / A_{22}, \\
& C_{22} \pm=\left(B_{22} \pm B_{2-2}\right) / A_{33}, \\
& C_{21} \pm=\left(F_{21} \pm F_{2-1}\right) /\left(E_{11} \pm E_{12} a_{2}\right),
\end{aligned}
$$

$$
\begin{aligned}
& B_{20}=-\frac{1}{4} i \tilde{f} \sin 2 \beta\left[-3(6 \pi / 5)^{1 / 2} j_{1}\right. \\
& \left.+\left(j_{1}-3 \xi j_{1}^{\prime}\right)\left(6^{1 / 2} a_{0}+2 a_{2} \cos 2 \alpha\right) / 7\right], \\
& B_{2, \pm 2}=-\frac{1}{4} i \text { 㚣 } \sin 2 \beta\left[3(\pi / 5)^{1 / 2} j_{1} e^{ \pm 2 i \alpha}\right. \\
& \left.+\left(j_{1}-3 \xi j_{1}^{\prime}\right)\left(a_{0} e^{ \pm 2 i \alpha}-6^{1 / 2} a_{2}\right) / 7\right] \text {, } \\
& F_{2, \pm 1}=\mp \frac{1}{2} i \tilde{r} \cos 2 \beta\left[3(\pi / 5)^{1 / 2} j_{1} e^{ \pm i \alpha}\right. \\
& \left.-\left(j_{1}-3 \xi j_{1}{ }^{\prime}\right)\left(a_{0} e^{ \pm i \alpha}+6^{1 / 2} a_{2} e^{\mp i \alpha}\right) / 7\right], \\
& 10\left(\frac{1}{3} \pi\right)^{1 / 2} A_{22}=X-a_{0} Y, \\
& 10\left(\frac{1}{3} \pi\right)^{1 / 2} A_{33}=X+a_{0} Y, \\
& 10\left(\frac{1}{3} \pi\right)^{1 / 2}\left(E_{11} \pm E_{12} a_{2}\right)=X-\frac{1}{2}\left(a_{0} \mp 6^{1 / 2} a_{2}\right) Y,
\end{aligned}
$$

where

$$
\begin{aligned}
& X=-3 j_{3}+2 j_{1}, \\
& Y=(5 / 7)(5 \pi)^{-1 / 2}\left[\left(6 j_{3}+j_{1}\right)+z\left(3 j_{3}{ }^{\prime}-2 j_{1}{ }^{\prime}\right)\right] .
\end{aligned}
$$

The argument of the spherical Bessel functions above is $z=k R_{0}$.

To begin with, we shall evaluate the $C_{L M}$ for an undeformed nucleus for which the different modes are degenerate. The electric field directions associated with diverse photon polarizations and directions are listed in Table I. We thus look for those choices of the Euler angles $\alpha, \beta$, and $\gamma$ which maximize the coefficients as given by (28). In comparison with the dipole resonance, for which the oscillations are in the direction of the electric field, the directions of the quadrupole oscillations are at an angle of $\frac{1}{4} \pi$ with respect to the direction of the electric field. The characteristics of the eigenmodes for the quadrupole oscillations are summarized in Table II.

The eigenmodes in Table II are those corresponding to the undeformed nucleus. A finite deformation will produce not only a change in the frequency of the oscillation but also a change in the direction of maximum displacement. Specifically, from (28) we find the results as summarized in Appendix A.

In order to calculate the eigenfrequencies and eigenenergies, we look for the zeros of the denominators in (A4). We have expressed these denominators in the form

where

$$
X+\Delta_{2} M^{\mu} Y=0,
$$

$$
\Delta_{20}{ }^{0}=-a_{0}, \quad \Delta_{22}^{ \pm}=a_{0}, \quad \Delta_{21}^{ \pm}=-\frac{1}{2}\left(a_{0} \mp 6^{1 / 2} a_{2}\right) .
$$

Since $-3 j_{3}(z)+2 j_{1}(z)=5 j_{2}{ }^{\prime}(z)$, we see that for the undeformed nucleus, the eigenfrequencies occur at the maxima and minima of $j_{2}(z)$. From $3 j_{3}(z)=2 j_{1}(z)$, there follows

$$
\cot z=\left(9-4 z^{2}\right) / z\left(9-z^{2}\right) .
$$

Then $C_{2 M}$ for $a_{\nu}=0$ has the poles given in Table III.

To calculate the eigenvalues for the deformed nucleus, we perform an expansion about the undeformed eigenvalues $\tilde{z}_{n}$.

Let

$$
z_{n}=\widetilde{z}_{n}+\Delta z_{n}
$$

TABLE II. The quadrupole modes and their corresponding directions of the maximum displacement of the proton fluid from equilibrium.

\begin{tabular}{cccc}
\hline \hline & \multicolumn{3}{c}{ Direction of } \\
maximum oscillation & $\begin{array}{c}\text { Periodicity } \\
\text { in } \varphi\end{array}$ \\
\hline$Y_{20}$ & $\theta$ & $\varphi$ & $\cdots$ \\
$Y_{21}+Y_{2-1}$ & 0 & $\cdots$ & $\pi$ \\
$Y_{21}-Y_{2-1}$ & $\frac{1}{4} \pi$ & $\frac{1}{2} \pi$ & 0 \\
$Y_{22}+Y_{2-2}$ & $\frac{1}{4} \pi$ & 0 & $\pi$ \\
$Y_{22}-Y_{2-2}$ & $\frac{1}{2} \pi$ & 0 & $\frac{1}{2} \pi$ \\
& $\frac{1}{2} \pi$ & $\frac{1}{4} \pi$ & $\frac{1}{2} \pi$ \\
\hline
\end{tabular}


Then

$$
\begin{aligned}
& X\left(z_{n}\right) \approx X^{\prime}\left(\widetilde{z}_{n}\right) \Delta z=\left(36 j_{4}-55 j_{2}+14 j_{0}\right)(1 / 21) \Delta z, \\
& Y\left(z_{n}\right) \approx Y\left(\widetilde{z}_{n}\right) .
\end{aligned}
$$

Thus

$$
\Delta z_{n}=-\Delta_{2 M^{\mu}} Y\left(\widetilde{z}_{n}\right) / X^{\prime}\left(\widetilde{z}_{n}\right)
$$

or

$z_{n}=\tilde{z}_{n}-15(5 \pi)^{-1 / 2} \Delta_{2 M}{ }^{\mu}\left(\frac{6 j_{3}+j_{1}}{36 j_{4}-55 j_{2}+14 j_{0}}-\frac{\tilde{z}_{n}}{21}\right)$.

Since

and

$$
k_{2 M}^{n \mu} R_{0}=z_{n}
$$

we then have

$$
k_{0}{ }^{n} R_{0}=\tilde{z}_{n}
$$

$$
\begin{aligned}
k_{2 M}{ }^{n \mu}=k_{0}{ }^{n} & {\left[1+15(5 \pi)^{-1 / 2} \Delta_{2 M^{\mu}}\right.} \\
& \left.\times\left(0.0476-\frac{6 j_{3}+j_{1}}{\widetilde{z}_{n}\left(36 j_{4}-55 j_{2}+14 j_{0}\right)}\right)\right] .
\end{aligned}
$$

For $n=1, \widetilde{z}_{n}=3.342$, we find

$$
k_{2 M}^{1 \mu}=k_{0}^{1}\left(1+0.284 \Delta_{2 M^{\mu}}\right) .
$$

Thus (dropping the superscript 1)

$$
\begin{aligned}
k_{20} & \equiv k_{20}{ }^{0}=k_{0}\left[1-0.284 a_{0}\right], \\
k_{2 \pm 1} & \equiv k_{21} \pm=k_{0}\left[1-0.142\left(a_{0} \mp 6^{1 / 2} a_{2}\right)\right], \\
k_{2 \pm 2} & \equiv k_{22} \pm=k_{0}\left[1+0.284 a_{0}\right] .
\end{aligned}
$$

Since the energies are proportional to the wave numbers $k_{2 M^{\mu}}$, the above relationships also hold true for the energies. Thus for $n=1$

$$
E_{2 \mu} \equiv E_{2 M^{\mu}}=E_{0}\left(1+0.284 \Delta_{2 M}{ }^{\mu}\right),
$$

where $E_{0}$ is dependent upon $A, N$, and $Z$ (see Sec. IIID). We note that $k_{20}$ and $k_{2 \pm 2}$ of Eq. (40) are in first-order independent of the nuclear asymmetry parameter $a_{2}$. This can be understood from Fig. 2 where the mode $\left(Y_{22}+Y_{2-2}\right)$ is shown for $a_{2}>0$ and $a_{2}<0$. [A similar situation exists for $\left(Y_{22}-Y_{2-2}\right)$.] In both cases this quadrupole mode "sees" a minor and a major axis simultaneously. Therefore, no linear dependence of the energy on $a_{2}$ can be expected for this mode.

\section{B. The Variational Calculation}

In order to obtain the energies of the quadrupole oscillations to second order in the deformation parameter $a_{0}$, we use a variational approach. The expansion method of the previous section becomes too involved to be practicable. The variational approach has been used by Inopin ${ }^{16}$ for the dipole case. A comparison of his results with the exact calculations of Danos $^{8}$ for the case of axial symmetry shows an agreement for the eigenvalues within $1 \%$.

${ }^{16}$ E. V. Inopin, Zh. Eksperim. i Teor. Fiz. 38, 992 (1960) [English transl.: Soviet Phys.-JETP 21, 714 (1960)].
TABLE III. Values of $\tilde{z}=k R_{0}$ for which the coefficients $C_{2 M}$ are maximized in the undeformed nucleus.

\begin{tabular}{cc}
\hline \hline$n$ & $\tilde{z}_{n}$ \\
\hline 1 & 3.342 \\
2 & 7.290 \\
3 & 10.614 \\
4 & 13.846 \\
$\vdots$ & $\vdots$ \\
$n$ & $\left(n+\frac{1}{2}\right) \pi$ \\
\hline
\end{tabular}

The technique used is described by Morse and Feshbach. ${ }^{17}$ For a trial solution $\eta_{t}$, which is dependent upon one or more parameters, the eigenvalue is given by

$$
k^{2}=\min \left\{\int\left|\nabla \eta_{t}\right|^{2} d \tau / \int\left|\eta_{t}\right|^{2} d \tau\right\}
$$

For trial solutions we use the wave functions for a sphere of volume equal to that of the considered nucleus. Thus

$$
\begin{aligned}
\eta_{20} & =j_{2}\left(k_{0} r\right) Y_{20}, \\
\eta_{2 \pm 1} & =j_{2}\left(k_{0} r\right)\left(Y_{21} \pm Y_{2-1}\right), \\
\eta_{2 \pm 2} & =j_{2}\left(k_{0} r\right)\left(Y_{22} \pm Y_{2-2}\right),
\end{aligned}
$$

with $k_{0}=3.34 / R_{0}$. To second order in $a_{0}$ and to first order in $a_{2}$, this calculation yields the results

$$
\begin{aligned}
k_{20} & =k_{0}\left[1-0.284 a_{0}-0.186 a_{0}^{2}\right], \\
k_{2 \pm 1} & =k_{0}\left[1-0.142\left(a_{0} \mp 6^{1 / 2} a_{2}\right)-0.077 a_{0}^{2}\right], \\
k_{2 \pm 2} & =k_{0}\left[1+0.284 a_{0}+0.048 a_{0}^{2}\right] .
\end{aligned}
$$

One observes that to first order in $a_{0}$ the expansion method and the variational calculation yield the same result for the energy. These results are indeed intimately

Fig. 2. Plane cut through the nuclear ellipsoid perpendicular to the main axis. The shaded areas show schematically the $\left(Y_{22}+Y_{2-2}\right)$ giant quadrupole mode. It is immediately apparent that this mode "sees" both axes (major and minor) simultaneously.

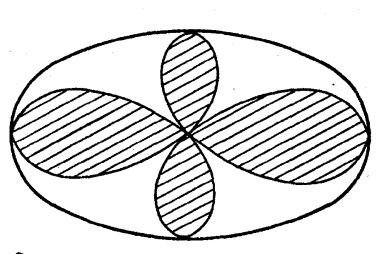
$a_{2}>0$

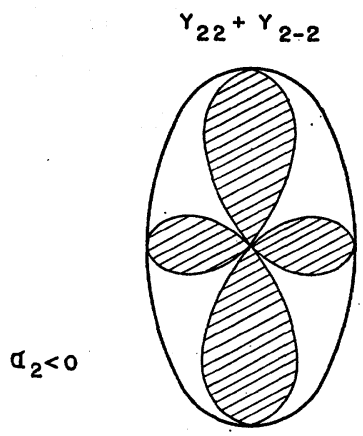

17 P. M. Morse and H. Feshbach, Methods of Theoretical Physics (McGraw-Hill Book Company, Inc., New York, 1953). 


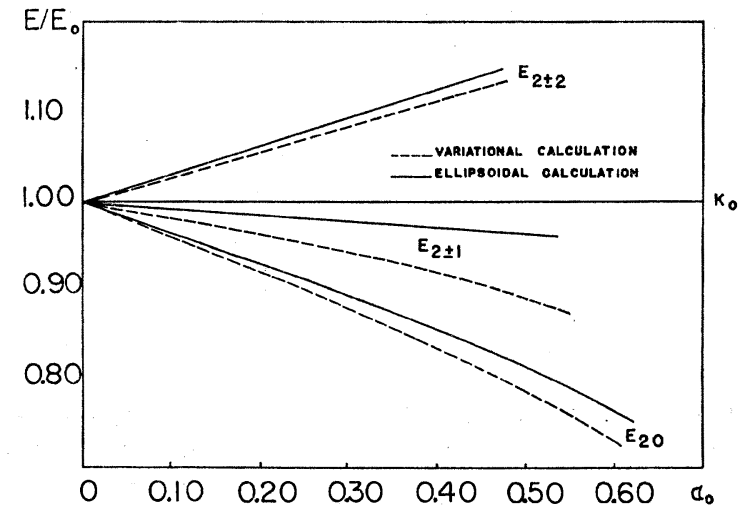

FIG. 3. Graph of the resonance energies of the various quadrupole modes in terms of the quadropole resonance energy for spherical nuclei $E_{0}$.

related. Consider a superposition of the trial solutions, Eq. (43)

$$
\eta=\sum_{m} \alpha_{m} \eta_{m} \text {. }
$$

Upon calculating Eq. (42) with this trial solution and minimizing with respect to the parameters $\alpha_{m}$, we would obtain a set of simultaneous equations. These equations are precisely the same as in the expansion method.

\section{The Exact Method}

Insofar as the nucleus may be treated as an ellipsoid of revolution, exact solutions to the Helmholtz equation (12) are obtainable. For small deformations the "quadrupole shape," defined by (14), and the shape of an ellipsoid of revolution are identical. However, already at the nuclear deformations corresponding to $a_{0} \approx 0.3$, the ellipsoidal and the quadrupole shapes differ somewhat. Hence, expanding the ellipsoidal shape in spherical harmonics, i.e., continuing to higher values of $l$, for such a deformation the term $a_{4} Y_{40}$ would have an amplitude $a_{4} \approx 0.1$. The consequences of this difference will become apparent below.

The exact solution is a linear superposition of spheroidal wave functions. These functions are solutions of Eq. (12) separated in spheroidal coordinates. For the prolate spheroid the transformations are given by ${ }^{18}$

where

$$
\begin{aligned}
& x=\frac{1}{2} \alpha\left(\xi^{2}-1\right)^{1 / 2}\left(1-\chi^{2}\right)^{1 / 2} \cos \varphi, \\
& y=\frac{1}{2} \alpha\left(\xi^{2}-1\right)^{1 / 2}\left(1-\chi^{2}\right)^{1 / 2} \sin \varphi, \\
& z=\frac{1}{2} \alpha \xi \chi
\end{aligned}
$$

$$
\begin{gathered}
1 \leq \xi<\infty, \\
-1 \leq \chi \leq 1, \\
0 \leq \varphi \leq 2 \pi .
\end{gathered}
$$

The foci of the ellipsoid (spheroid) are $z= \pm \frac{1}{2} \alpha$, and $x, y, z$ are the rectangular coordinates. In spheroidal

${ }^{18}$ F. J. Corbato et al., Spheroidal Wave Functions (John Wiley \& Sons, Inc., New York, 1956). coordinates a solution of (2) is then of the form

$$
\eta=\Phi(\varphi) S(\chi) J(\xi),
$$

where $J(\xi)$ obeys the ordinary differential equation in $\xi$

$$
\frac{d}{d \xi}\left[\left(\xi^{2}-1\right) \frac{d J^{-}}{d \xi}\right]-\left[A-h^{2} \xi^{2}+\frac{m^{2}}{\xi^{2}-1}\right] J=0 .
$$

$A$ and $m$ are separation constants and

$$
h=\frac{1}{2} k \alpha \text {. }
$$

Expressed in spheroidal coordinates, the surface of an ellipsoid is a surface of constant $\xi$ for a certain value of the parameter $h$, and the normal gradient operation is simply the differentiation with respect to $\xi$. The spheroidal function can be expanded in spherical Bessel functions. In the notation of Stratton et $a l .{ }^{18}$ this expansion is

$$
\begin{aligned}
J(\xi)=j e_{m l}(h, \xi)=\xi^{-m} & \left(\xi^{2}-1\right)^{m / 2} \\
& \times \sum_{n}{ }^{\prime} a_{n}(h \mid m l) j_{n+m}(h \xi) .
\end{aligned}
$$

The coefficients $a_{n}(h \mid m l)$ are given in Ref. 18. The prime on the summation sign indicates that the expansion contains only even or odd $n$. Then

$$
\hat{n} \cdot \nabla \eta=\sum_{l=0}^{\infty} \sum_{m=0}^{l} F_{m l}(h, \varphi, \chi)(\partial / \partial \xi) j e_{m l}(h, \xi) .
$$

By Eq. (51) we have

$$
\begin{aligned}
& (\partial / \partial \xi) j e_{m l}(h, \xi) \\
& =m\left(\xi^{2}-1\right)^{(m / 2)-1} \xi^{-m-1} \sum_{n}{ }^{\prime} a_{n}(h \mid m l) j_{n+m}(h \xi) \\
& \quad+\left(\xi^{2}-1\right)^{m / 2} \xi^{-m} \sum_{n}{ }^{\prime} \frac{a_{n}(h \mid m l)}{2(n+m)+1} \\
& \quad \times\left[(n+m) j_{n+m-1}(h \xi)\right. \\
& \left.\quad-(n+m+1) j_{n+m+1}(h \xi)\right] .
\end{aligned}
$$

The semimajor and semiminor axes of the ellipsoid are given by

$$
\begin{aligned}
& a=R_{0}\left[1+(5 / 4 \pi)^{1 / 2} a_{0}\right] \\
& b=R_{0}\left[1-\frac{1}{2}(5 / 4 \pi)^{1 / 2} a_{0}\right] .
\end{aligned}
$$

In terms of the deformation parameter $a_{0}$, the parameter $h$ is given by

$$
h=\frac{1}{2} k R_{0}\left[15(5 \pi)^{-1 / 2} a_{0}+(15 / 8 \pi) a_{0}^{2}\right]^{1 / 2} .
$$

Furthermore, $a_{0}$ and the coordinate $\xi$ are related by the quadratic equation

$$
0.299\left(\xi^{2}-1.333\right) a_{0}^{2}+1.893\left(\xi^{2}-0.667\right) a_{0}-1=0 \text {. }
$$

We now consider the quadrupole term, viz. $l=2$ in the expansion (52). The boundary condition yields

$$
(\partial / \partial \xi) j e_{m l}(h, \xi)=0 \text {. }
$$


These equations corresponding to $m=0,1,2$ are given in Appendix A. By solving these equations, viz. (A8), (A9), (A10), and using (56) and (57), one obtains the curves (Fig. 3) relating the parameters $a_{0}$ and the eigenvalues $E_{2}$.

\section{The Resonant Energies for Spherical Nuclei}

In Fig. 3 we give the resonant energies of the fundamental quadrupole modes in terms of the energy of the degenerate modes of the undeformed nucleus. This energy, $E_{0}$, is given by

$$
E_{0}=\hbar \omega_{0}=\hbar k_{0} u
$$

where $u$ is the velocity of "second sound" in the nucleus. Together with (6), the energy is then

$$
E_{0}=\left(\hbar \tilde{z}_{1} / R_{0}\right)\left[\left(8 \kappa / M^{*}\right)\left(N Z / A^{2}\right)\right]^{1 / 2} .
$$

If we use $4 N Z / A^{2}=1, \kappa=20 \mathrm{MeV}, R_{0}=1.35 A^{1 / 3} \mathrm{fm}$, $\widetilde{z}_{1}=3.34$, and $\alpha=0.5$ for the effective mass, Eq. (7), then

$$
E_{0} \approx 125 A^{-1 / 3} \text {. }
$$

This value of $E_{0}$ is affected by several sources of uncertainty. First, the value of the symmetry energy constant is uncertain by about $\pm 10 \%$. Second, the diffuseness of the nuclear shape also affects the resonance energies. Both effects have to be known to determine the effective mass. However, since the same effects are present in the dipole as in the quadrupole resonance, they will presumably have little influence on the ratio

$$
\frac{E_{\text {quadrupole }}}{E_{\text {dipole }}}=1.6 .
$$

\section{THE QUADRUPOLE OPERATOR}

The electric quadrupole moment operator in the laboratory system, $\hat{Q}_{\mu}$, is obtained by transforming the quadrupole operator in the intrinsic system $Q_{\nu}$ with the rotation matrices

$$
\hat{Q}_{\mu}=\sum_{\nu=-2}^{2} D_{\mu \nu}{ }^{(2)}\left(\theta_{j}\right) Q_{\nu}
$$

The operator in the intrinsic system is defined as

$$
Q_{\nu}=\int \rho_{p} r^{2} Y_{2 \nu} d V
$$

The integral is taken over the nuclear volume with the proton distribution $\rho_{p}$ given by ${ }^{1}$

$$
\rho_{p}=\rho_{p}(0)\left[1+\sum_{\mu=-2}^{2} \tau_{\mu} F_{\mu} \eta_{\mu}(\mathbf{r})\right]
$$

Here $\rho_{p}(0)$ is the equilibrium proton density. The $F_{\mu}$ are normalization factors. $\tau_{\mu}$ characterizes the amplitude of the quadrupole oscillation associated with the mode $\mu$. If we assume harmonic quadrupole oscillations, then the potential energy assumes the form

$$
V(\tau)=\sum_{\mu} \frac{1}{2} C_{\mu}\left|\tau_{\mu}\right|^{2} .
$$

The $C_{\mu}$ are constants which are to be determined.

In the hydrodynamic model the potential energy is given by ${ }^{1}$

$$
V(\tau)=\kappa \int\left|\rho_{0}-2 \rho_{p}\right|^{2} / \rho_{0} d V+b .
$$

$\rho_{0}$ is the nucleon density. The integration is again taken over the nuclear volume. $b$ is a constant. Equating Eqs. (66) and (67), we have

$$
C_{\mu}=\left[8 \kappa \rho_{p}^{2}(0)\left|F_{\mu}\right|^{2} / \rho_{0}\right] \int\left|\eta_{\mu}\right|^{2} d V
$$

Upon calculation of the integrals with the wave functions

$$
\begin{aligned}
\eta_{20} & =j_{2}\left(k_{20} r\right) Y_{20}, \\
\eta_{2 \pm 1} & =(1 / \sqrt{2}) j_{2}\left(k_{2 \pm 1} r\right)\left(Y_{21} \pm Y_{2-1}\right), \\
\eta_{2 \pm 2} & =(1 / \sqrt{2}) j_{2}\left(k_{2 \pm 2} r\right)\left(Y_{22} \pm Y_{2-2}\right),
\end{aligned}
$$

we obtain the expressions for the $C_{\mu}$, which are given in Appendix B.

Let us now define

$$
Q_{\nu}^{\prime}=\int \rho_{p}(0) r^{2} Y_{2 \nu} d V
$$

This is the intrinsic quadrupole operator resulting only from the nonspherical shape of the nucleus. It is nonzero already for $\eta=0$, i.e., for a uniform proton distribution. We find

$$
\begin{aligned}
Q_{0} & =R_{0}^{5} \rho_{p}(0)\left[a_{0}+(10 / 7)(5 \pi)^{-1 / 2} a_{0}^{2}\right], \\
Q_{ \pm 1} & =0 \\
Q_{ \pm 2}{ }^{\prime} & =R_{0}^{5} \rho_{p}(0) a_{2} .
\end{aligned}
$$

We now introduce the annihilation and creation operators for the quadrupole oscillations, $q_{\nu}$ and $q_{\nu}^{\dagger}$, respectively:

$$
\sum_{\mu}\left(\frac{1}{2} B_{\mu}\left|\dot{\tau}_{\mu}\right|^{2}+\frac{1}{2} C_{\mu}\left|\tau_{\mu}\right|^{2}\right) \equiv \sum_{\mu} \hbar \omega_{\mu} q_{\mu}^{\dagger} q_{\mu}
$$

which, actually, is also a defining equation for the mass parameter $B$. The amplitudes $\tau$ thus are related to the annihilation and creation operators by

$$
\tau_{\mu}=\left(\hbar \omega_{\mu} / 2 C_{\mu}\right)^{1 / 2}\left[q_{\mu}^{\dagger}+(-)^{\mu} q_{-\mu}\right]
$$

and $\omega_{\mu}=k_{2 \mu} u$. This relation insures that $\tau_{\mu}{ }^{*}=(-)^{\mu} \tau_{-\mu}$. A change in notation with respect to previous calculations ${ }^{1,2}$ has been introduced in order to conform to the standard phase conventions. We list the expressions for the intrinsic quadrupole operators in Appendix B. From these formulas, Eq. (B2), one can easily give an estimate of the induced quadrupole moments, which are $Q_{\mu}-Q_{\mu}{ }^{\prime}$. We find $Q_{0}-Q_{0}{ }^{\prime} \approx 3 \mathrm{~b}$ while $Q_{0}{ }^{\prime} \approx 5 \mathrm{~b}$. Thus 
the induced giant resonance quadrupole moments are of the same order of magnitude as the intrinsic quadrupole moments of the nuclear shape.

\section{PHOTON ABSORPTION CROSS SECTION}

The explicit expression we use for the total photon absorption cross section is ${ }^{1,19,20}$

$$
\sigma^{n}(E)=\frac{2 \pi}{\hbar c} \frac{1}{\mathcal{E}_{0}^{2}}\left|\left\langle H_{Q}\right\rangle_{n 0}\right|^{2} \frac{\Gamma E^{n 0}}{\left(E^{n 0}-E\right)^{2}+\frac{1}{4} \Gamma^{2}} .
$$

$H_{Q}$ is the quadrupole interaction and $\Gamma$ is the width of the resonance.

For a localized charge distribution $\rho(\mathbf{r})$ placed in an external potential $\Phi(\mathbf{r})$, the electrostatic interaction of the system is

$$
W=\int \rho(\mathbf{r}) \Phi(\mathbf{r}) d V
$$

Expanding the potential around an origin chosen at the center of the nucleus, we have

$$
W=q \Phi(0)-\mathbf{d} \cdot \mathcal{E}-\frac{1}{6} \sum_{i, j} Q_{i j} \frac{2 \mathcal{E}_{j}(0)}{2 x_{i}}+\cdots .
$$

Here $q$ and $\mathbf{d}$ are the total charge and the dipole moment, respectively. $Q_{i j}$ is the quadrupole moment given by

$$
Q_{i j}=e \int\left(3 x_{i} x_{j}-r^{2} \delta_{i j}\right) \rho(\mathbf{r}) d V .
$$

Upon comparison of Eq. (77) with Eq. (64), we obtain the explicit expressions for the $Q_{i j}$ as listed in Appendix B. The $Q_{\mu}{ }^{\prime}$ do not have to be included since the matrix element of these operators between the ground state and a giant $-E 2$-state vanishes.

To calculate $\partial \mathcal{E}_{j}(0) / \partial x_{i}$ we consider an incident plane wave traveling in the direction $\mathbf{s}$ with wave number $s$ and frequency $\omega$ with the electric field polarized in the direction $\hat{u}$

$$
\boldsymbol{\varepsilon}=\hat{u} \mathcal{E}_{0} e^{i(\mathbf{s} \cdot \mathbf{r}-\omega t)},
$$

where $|\hat{\imath}|=1$. Writing the Cartesian coordinates as

$$
\begin{aligned}
& \mathbf{s}=s_{1} \hat{x}+s_{2} \hat{y}+s_{3} \hat{z}, \\
& u=u_{1} \hat{x}+u_{2} \hat{y}+u_{3} \hat{z},
\end{aligned}
$$

the gradient of $\varepsilon$ evaluated at the origin becomes

$$
\boldsymbol{\nabla} \boldsymbol{\varepsilon}(0)=i s \mathcal{E}_{0}[M] e^{-i \omega t} .
$$

$[M]$ is a $(3 \times 3)$ matrix with components

$$
M_{i j}=s_{i} u_{j} / s .
$$

${ }^{19}$ S. Flugge, Z. Naturforsch. 1, 121 (1946). ${ }^{20}$ S. Flugge, Z. Naturforsch. 3a, 97 (1948).
Since

$$
H_{Q}=\frac{1}{6} \sum_{i, j} Q_{i j} \frac{\partial \mathcal{E}_{j}(0)}{\partial x_{i}},
$$

the elements of the matrix may be expressed as

$$
\left\langle\left|H_{Q}\right|\right\rangle_{n 0}=\frac{1}{6} i s \mathcal{E}_{0} e^{-i \omega t}\left\langle\left|\sum_{i, j} Q_{i j} M_{i j}\right|\right\rangle_{n 0} .
$$

Using $s=\omega / c=E / \hbar c$, we find for the cross section

$$
\sigma_{\mu}{ }^{n}(E)=2 \pi \frac{E^{2} E_{2 \mu}{ }^{n 0}}{(\hbar c)^{3}}\left|\langle|Q|\rangle_{n 0}\right|^{2} \frac{\Gamma}{\left(E_{2 \mu}{ }^{n 0}-E\right)^{2}+\frac{1}{4} \Gamma^{2}},
$$

where we have denoted the mode by the subscript $\mu$, and the energy level by $n$. We have also defined

$$
Q=\frac{1}{6} \sum_{i, j} Q_{i j} M_{i j}
$$

We now calculate the integrated cross section and the quadrupole sum rule. Following Khokhlov, ${ }^{21}$ we have the sum rule

$$
\begin{aligned}
\int_{0}^{\infty} \frac{\sigma_{\mu}(E)}{E^{2}} d E & =4 \pi^{2}(\hbar c)^{-3}\left[-\frac{1}{2} i \hbar\langle Q \dot{Q}-\dot{Q} Q\rangle_{00}\right] \\
& =\frac{1}{5} \pi^{2}\left(e^{2} / \hbar c\right)\left(M^{*} c^{2}\right)^{-1} Z R_{2 \mu}{ }^{2},
\end{aligned}
$$

with $\sigma_{\mu}(E)=\sum_{n} \sigma_{\mu}{ }^{n}(E)$. In the limit as $\Gamma \rightarrow 0$ the function

$$
\frac{\Gamma}{\left(E_{2 \mu}{ }^{n}-E\right)^{2}+\frac{1}{4} \Gamma^{2}}
$$

behaves as a Dirac delta function, $2 \pi \delta\left(E-E_{2 \mu}{ }^{n}\right)$, upon integration over the energy from 0 to $\infty$. To apply this sum rule, we have to include a factor $N / A$ which has its origin in the fact that the hydrodynamic model contains only the "second sound" part of the sum rule in which the protons and the neutrons move in opposite fashion. For example, the contributions of surface vibrations where protons and neutrons move together have to be excluded here. We now define the E2 oscillator strengths by

$$
\sum_{n} f_{2 \mu}{ }^{n 0}=\left(N Z / A^{2}\right) R_{2 \mu}{ }^{2},
$$

TABLE IV. Oscillator strengths and cross sections for the quadrupole modes of ${ }_{68} \mathrm{Er}^{166}$ for the special orientations of Table $\mathrm{I}$. $E_{2 \mu}$ is obtained from the variational calculation. $W_{\mu}=E^{2} \Gamma /$ $\left[\left(E_{2 \mu}-E\right)^{2}+\Gamma^{2} / 4\right]$.

\begin{tabular}{cccc}
\hline \multicolumn{1}{c}{$\mu$} & ${ }_{2} f_{\mu}{ }^{10} /\left(R_{0}{ }^{2} N Z / A\right)$ & $\begin{array}{c}E_{2 \mu} \\
(\mathrm{MeV})\end{array}$ & $\begin{array}{c}\sigma_{\mu}{ }^{2}(E) / W_{\mu} \\
(\mathrm{mb} / \mathrm{MeV})\end{array}$ \\
\hline 0 & 0.866 & 20.3 & $5.54 \times 10^{-2}$ \\
+1 & 0.890 & 22.1 & $5.69 \times 10^{-2}$ \\
-1 & 0.965 & 21.3 & $6.17 \times 10^{-2}$ \\
\pm 2 & 0.157 & 25.2 & $1.00 \times 10^{-2}$ \\
\hline
\end{tabular}

${ }^{21}$ K. Y. Khokhlov, Zh. Eksperim. i Teor. Fiz. 32, 124 (1957) [English transl.: Soviet Phys.-JETP 5, 88 (1957)]. 
TABLE V. Excitation energies for the quadrupole modes in heavy deformed nuclei. The values of $E_{2}$ are calculated using the variational technique, except for the last column. Comparison of the mean value of $E_{2+1}$ and $E_{2-1}$ with the value obtained by the ellipsoidal treatment (Exact) indicates that in some cases there is a difference of as much as $1 \mathrm{MeV}$ in the eigenenergies."

\begin{tabular}{|c|c|c|c|c|c|c|c|c|c|}
\hline Element & $a_{0}$ & $a_{2} / a_{0}$ & $\begin{array}{c}E_{0} \\
(\mathrm{MeV})\end{array}$ & $\begin{array}{c}E_{20} \\
(\mathrm{MeV})\end{array}$ & $\begin{array}{c}E_{2 \pm 2} \\
(\mathrm{MeV})\end{array}$ & $\begin{array}{c}E_{2+1} \\
(\mathrm{MeV})\end{array}$ & $\begin{array}{c}E_{2-1} \\
(\mathrm{MeV})\end{array}$ & $\begin{array}{c}\text { Mean } \\
E_{2 \pm 1} \\
(\mathrm{MeV})\end{array}$ & $\begin{array}{c}\text { Exact } \\
E_{2 \pm 1} \\
(\mathrm{MeV})\end{array}$ \\
\hline${ }_{64} \mathrm{Gd}^{154}$ & 0.33 & 0.162 & 23.5 & 20.8 & 25.9 & 22.6 & 21.8 & 22.2 & 23.0 \\
\hline${ }_{64} \mathrm{Gd}^{156}$ & 0.41 & 0.132 & 23.4 & 20.0 & 26.3 & 22.1 & 21.3 & 21.7 & 22.8 \\
\hline${ }_{64} \mathrm{Gd}^{158}$ & 0.46 & 0.125 & 23.3 & 19.4 & 26.6 & 21.7 & 20.7 & 21.2 & 22.7 \\
\hline${ }_{64} \mathrm{Gd}^{160}$ & 0.47 & 0.134 & 23.2 & 19.2 & 26.5 & 21.6 & 20.6 & 21.1 & 22.6 \\
\hline${ }_{68} \mathrm{Er}^{166}$ & 0.33 & 0.155 & 22.9 & 20.3 & 25.2 & 22.1 & 21.3 & 21.7 & 22.4 \\
\hline${ }_{68} \mathrm{Er}^{168}$ & 0.33 & 0.150 & 22.8 & 20.2 & 25.1 & 22.0 & 21.2 & 21.6 & 22.3 \\
\hline${ }_{90} \mathrm{Th}^{230}$ & 0.23 & 0 & 20.6 & 19.0 & 22.0 & 19.9 & 19.9 & 19.9 & 20.3 \\
\hline${ }_{90} \mathrm{Th}^{232}$ & 0.25 & 0 & 20.5 & 18.8 & 22.0 & 19.7 & 19.7 & 19.7 & 20.1 \\
\hline
\end{tabular}
a In these calculations we have used $E_{0}=125 A^{-1 / 3}, E_{20}=E_{0}\left[1-0.284 a_{0}-0.186 a_{0}^{2}\right], E_{2_{ \pm 1}}=E_{0}\left[1-0.142\left(a_{0} \pm(6)^{1 / 2} a_{2}\right)-0.077 a_{0}^{2}\right]$, and $E_{2 \pm 2}=E_{0}\left[1+0.284 a_{0}\right.$
$\left.+0.048 a_{0}^{2}\right]$.

then comparison of (84) and (86) shows that

$$
f_{2 \mu}{ }^{n 0}=20 M^{*} c^{2}(\hbar c)^{-3}\left(\hbar c / e^{2}\right) E_{2 \mu}{ }^{n 0}\left|\langle|Q|\rangle_{n 0}\right|^{2} \text {. }
$$

Let us define [see Eq. (B3)]

Since

$$
Q_{i j}=0.492 e\left(\rho_{0} R_{0}^{5} / \kappa\right)^{1 / 2} N_{i j}
$$

then

$$
\rho_{0}=A / \frac{4}{3} \pi R_{0}^{3} \text {, }
$$

$f_{2 \mu}{ }^{n 0}=0.26\left(k_{2 \mu}{ }^{n}\right)^{2}\left(\hbar \omega_{\mu}{ }^{n}\right)^{-1}\left(R_{0} / R_{2 \mu}\right)^{2}$

$$
\times\left|\left\langle\left|\sum_{i, j} M_{i j} N_{i j}\right|\right\rangle_{n 0}\right|^{2}(N Z / A) R_{2 \mu}^{2} \text {. }
$$

In terms of the oscillator strength, the photon absorption cross section for the region of the giant quadrupole resonance is

$$
\sigma_{\mu}^{1}(E)=\frac{\pi}{10} \frac{1}{M^{*} c^{2}} \frac{e^{2}}{h c} f_{2 \mu}^{10} \frac{E \Gamma}{\left(E_{2 \mu}{ }^{10}-E\right)^{2}+\frac{1}{4} \Gamma^{2}} .
$$

\section{CALCULATION OF THE OSCILLATOR STRENGTHS AND TOTAL ABSORPTION CROSS SECTION}

We begin by considering the case of a spherical nucleus. Then $k_{2 \mu}{ }^{1}=k_{0}, \omega_{\mu}{ }^{1}=\omega_{0}$ and $R_{2 \mu}=R_{0}$. Equation (90) for $n=1$ then becomes

$$
f_{2 \mu}^{10}=0.26\left(k_{0}{ }^{2} / \hbar \omega\right)\left|\left\langle\left|\sum_{i j} M_{i j} N_{i j}\right|\right\rangle_{10}\right|^{2}(N Z / A) R_{0}^{2} .
$$

Let $\alpha$ and $\beta$ be the Euler angles defining the direction of the incident photon with wave vector $\mathrm{s}$ in the intrinsic coordinate system. We consider the four separate cases as given in Table I. These cases correspond to different orientations of the nucleus with respect to the incident photon beam. The explicit formulas are given in Appendix B.

We now turn to deformed nuclei. The cross section for the mode $\mu$ is

$$
\sigma_{\mu}{ }^{1}(E)=3.66 \times 10^{-5} f_{2 \mu}{ }^{10} \frac{E^{2} \Gamma}{\left(E_{2 \mu}{ }^{10}-E\right)^{2}+\frac{1}{4} \Gamma^{2}},
$$

where $f_{2}{ }^{10}$ is in $10^{-26} \mathrm{~cm}^{2}, \Gamma$ and $E$ in $\mathrm{MeV}$, and $\sigma_{\mu}{ }^{1}(E)$ in $\mathrm{mb}$. Let us consider as an example a nucleus with the parameters of ${ }_{68} \mathrm{Er}^{166}$. From low-energy data $a_{0}=0.33$ and, effectively, $a_{2}=0.051$. If we assume $M^{*}=0.57 M$ and $R_{0}=1.2 A^{1 / 3} \mathrm{fm}=6.6 \mathrm{fm}$, then $E_{0}=22.9$ $\mathrm{MeV}$. The results are summarized in Table IV and are for the cases listed in Appendix B. The total cross section for the energy $E$ is given by

$$
\sigma^{1}(E)=\sum_{\mu} \sigma_{\mu}^{1}(E)
$$

\section{RESULTS AND DISCUSSION}

In a spherical nucleus the five quadrupole modes of oscillation are degenerate. However, they are not degenerate in a deformed, triaxial nucleus, and they have, to a good approximation, angular distributions proportional to $Y_{20}, \quad\left(Y_{21} \pm Y_{2-1}\right), \quad\left(Y_{22} \pm Y_{2-2}\right)$. Of particular interest is the result that the eigenfrequencies of the $\left(Y_{21} \pm Y_{2-1}\right)$-distributions depend strongly on the form of the deformed nucleus, ${ }^{6}$ e.g., whether it is an ellipsoidal shape or a quadrupole shape where $R=R_{0}\left(1+\alpha_{2 \mu} Y_{2 \mu}\right)$ (Fig. 3). Since the $\left(Y_{21} \pm Y_{2-1}\right)$ modes are peaked at $\theta \approx n \pi / 4$, we compare the radial distances of the ellipsoidal shape and the quadrupole shape in this direction, assuming axial symmetry. We have for an ellipsoid

$$
R_{\mathrm{el}}^{2}=2 a^{2} b^{2} /\left(a^{2}+b^{2}\right)
$$

where $a$ and $b$ are the semimajor and semiminor axes of the ellipsoid, respectively. We then find

$$
R_{\mathrm{el}}=R_{0}\left[1+\frac{1}{4}(5 / 4 \pi)^{1 / 2} a_{0}-(15 / 16 \pi) a_{0}^{2}+\cdots\right] .
$$

For the quadrupole shape we have

$$
R_{\mathrm{q}}=R_{0}\left[1+\frac{1}{4}(5 / 4 \pi)^{1 / 2} a_{0}\right] .
$$

Hence, the ratio of the change in the radial distance at $\pi / 4$ as a function of $a_{0}$ is

$$
\Delta R_{\mathrm{el}} / \Delta R_{\mathrm{q}} \approx 1-3(5 / 4 \pi)^{1 / 2} a_{0} .
$$


With $a_{0} \approx \frac{1}{3}$, we then find

$$
\left(\Delta R_{\mathrm{el}} / \Delta R_{\mathrm{q}}\right) \approx\left(\Delta E_{\mathrm{el}} / \Delta E_{\mathrm{q}}\right),
$$

where $\Delta E_{\mathrm{q}}$ and $\Delta E_{\mathrm{el}}$ are the shift of the mean resonant energies of the modes $\left(Y_{21} \pm Y_{2-1}\right)$ in the cases of quadrupole shapes and ellipsoidal shapes, respectively. The difference in the resonant energies for these modes is thus significant enough to shift the position of the maximum cross section by as much as $1 \mathrm{MeV}$. This difference is shown explicitly for heavy nuclei in Table $\mathrm{V}$ by comparison of the variational calculation (mean) and the ellipsoidal calculation (exact). As $a_{0}$ becomes large, (98) becomes inaccurate since the direction of maximum oscillation for these modes is then at an angle less than $\frac{1}{4} \pi$.

We now make a few remarks about the magnitude of the errors involved in the calculation and the validity of the model. As noted in Sec. IIIB, the energies as calculated by the variational method could be improved by considering the trial wave functions as a sum of spherical harmonics and varying the coefficients to obtain the closest approximation to the exact wave function and its corresponding eigenvalues. This procedure was followed in certain modes, but the values of the eigenenergies were decreased by less than $0.5 \%$.

The calculation of the resonant energy of the undeformed nucleus in Sec. IIID depends on the values of $\kappa$ and $M^{*}$. The uncertainties in these values result in an uncertainty of the resonant energy of about $20 \%$. However, this uncertainty will have a very small effect on the shape of the cross section and the splitting of the resonance for a deformed nucleus. The value of $E_{0}$ for the quadrupole resonance can be expected to appear at $1.6 E_{0}{ }^{\prime}$, where $E_{0}{ }^{\prime}$ is the position of the giant dipole resonance. Therefore, from the knowledge of the latter, we can precisely determine the position of the quadrupole resonance. Another significant change in the resonant energies would be expected to result from a consideration of a dynamic theory. We have here neglected the coupling of the quadrupole oscillations with surface vibrations $(\sim 1 \mathrm{MeV})$, rotational modes $(\sim 100 \mathrm{keV})$, and single particle motions $(\sim 500 \mathrm{keV})$. The inclusion of these interactions for a dynamic treatment of the problem should constitute the next step in the theory of the giant quadrupole resonance.

The experimental problems involved in the detection of the giant quadrupole resonance are considerable. In the multipole expansion of the radiation field, the quadrupole term is about $s R$ times as big as the dipole term. For $s R \approx 0.7$ the ratio of the cross sections would then be about 0.50 since the cross section is proportional to the square of the matrix element. Furthermore, the tail of the dipole resonance in the quadrupole resonance region is still $10 \%$ to $20 \%$ of its maximum value. If direct photon absorption experiments were performed, it would be necessary to subtract the dipole contribution in the energy region under consideration to obtain the quadrupole resonance. A more sensitive experimental technique perhaps might be inelastic electron scattering at an angle for which the dipole contribution to the scattering has a minimum; the quadrupole contribution would not be depressed at that angle. The drawback here would be the difficulty in the subtraction of the radiation tails associated with the inelastic scattering events leading to all nuclear levels which have lower excitation energies than the desired level. In balance, none of the possible experiments are simple in interpretation.

\section{APPENDIX A}

We list here several long formulas which were obtained in the solution of the Helmholtz equation in Sec. III.

In terms of spherical unit vectors, the expansion of $\nabla \eta(\mathbf{r})$, Eq. (8), is

$$
\begin{aligned}
\nabla \eta(\mathbf{r})= & \sum_{L, M, \mu} C_{L M} k\left\{[(L+1) /(2 L+1)]^{1 / 2} j_{L+1}(k r)\right. \\
& \times(1, L+1, L \mid-\mu, M+\mu, M) Y_{L+1, M+\mu} \\
& +[L /(2 L+1)]^{1 / 2} j_{L-1}(k r) \\
& \left.\times(1, L-1, L \mid-\mu, M-\mu, M) Y_{L-1, M+\mu}\right\} \xi_{-\mu} .
\end{aligned}
$$

The expansion coefficients of the normal unit vectors in terms of the spherical unit vectors, Eq. (13), is

$$
\begin{gathered}
\alpha_{ \pm 1}=-\left[1+(5 \pi)^{-1 / 2} a_{0}\right] Y_{1 \mp 1}-9(70 \pi)^{-1 / 2} a_{0} Y_{3 \mp 1} \\
-(6 / 5 \pi)^{1 / 2} a_{2} Y_{1 \pm 1}-\frac{3}{2}(3 / 35 \pi)^{1 / 2} Y_{3 \pm 1} \\
-9(28 \pi)^{-1 / 2} a_{2} Y_{3 \mp 3} \\
\alpha_{0}=\left[1-2(5 \pi)^{-1 / 2} a_{0}\right] Y_{10}+9(3 / 140 \pi)^{1 / 2} Y_{30} \\
+\frac{3}{2}(3 / 7 \pi)^{1 / 2} a_{2}\left(Y_{32}+Y_{3-2}\right) .
\end{gathered}
$$

The expression for $\left.\hat{n} \cdot \nabla \eta\right|_{r=R}$, after expansion up to terms linear in $a_{0}$ and $a_{2}$, is of the form

$$
\begin{aligned}
\left.\hat{n} \cdot \nabla \eta\right|_{r=R}= & \sum_{L, M} C_{L M} k\left[W_{L M} Y_{L M}+W_{L+2, M} Y_{L+2, M}\right. \\
& +W_{L-2, M} Y_{L-2, M}+W_{L+4, M} Y_{L+4, M} \\
& +W_{L-4, M} Y_{L-4, M}+W_{L, M+2} Y_{L, M+2} \\
& \left.+W_{L, M-2} Y_{L, M-2}\right]
\end{aligned}
$$

where the coefficients $W_{L M}$ are given in Ref. 22. They are too long to be listed here.

The excited modes and the corresponding quadrupole expansion coefficients, using the abbreviations $C_{L M^{ \pm}}$ $=C_{L M} \pm C_{L-M}$, are

$$
\begin{aligned}
& \begin{array}{l}
\alpha=0, \beta=0, \gamma=0 \\
C_{20}=C_{21}{ }^{+}=C_{22}{ }^{+}=C_{22}{ }^{-}=0, \\
C_{21}{ }^{-}=i \uparrow\left[42(\pi / 5)^{1 / 2} j_{1}-\left(j_{1}-3 \zeta j_{1}{ }^{\prime}\right)\left(a_{0}+6^{1 / 2} a_{2}\right)\right] / \\
{\left[14\left(E_{11}-E_{12} a_{2}\right)\right] .}
\end{array}
\end{aligned}
$$

${ }^{22}$ C. B. Kohr, thesis, University of Maryland, 1965 (unpublished). 


$$
\begin{aligned}
& \alpha=\frac{1}{2} \pi, \beta=0, \gamma=0 \\
& C_{20}=C_{21}{ }^{-}=C_{22}{ }^{+}=C_{22}{ }^{-}=0 \text {, } \\
& C_{21}{ }^{+}=i \uparrow\left[42(\pi / 5)^{1 / 2} j_{1}+\left(j_{1}-3 \zeta j_{1}{ }^{\prime}\right)\left(a_{0}-6^{1 / 2} a_{2}\right)\right] / \\
& \alpha=0, \beta=\frac{1}{4} \pi, \gamma=0 \\
& {\left[14\left(E_{11}+E_{12} a_{2}\right)\right] \text {. }} \\
& C_{21}{ }^{+}=C_{21}{ }^{-}=C_{22}{ }^{-}=0 \text {, } \\
& C_{20}=i \tilde{\boldsymbol{f}} /\left(28 A_{22}\right)\left[21(6 \pi / 5)^{1 / 2} j_{1}\right. \\
& \left.-\left(j_{1}-3 \zeta j_{1}^{\prime}\right)\left(6^{1 / 2} a_{0}+2 a_{2}\right)\right], \\
& C_{22}{ }^{+}=-i \mathfrak{\uparrow} /\left(14 A_{33}\right)\left[21(\pi / 5)^{1 / 2} j_{1}\right. \\
& \left.+\left(j_{1}-3 \zeta j_{1}{ }^{\prime}\right)\left(a_{0}-6^{1 / 2} a_{2}\right)\right] \text {. } \\
& \left.-\left(j_{1}-3 \zeta j_{1}{ }^{\prime}\right) 6^{1 / 2} a_{0}\right],
\end{aligned}
$$

For the spheroidal (exact) calculation the eigenvalue equations resulting from the boundary condition, Eq. (58), are

$$
\begin{aligned}
& \begin{array}{l}
m=0 \\
\sum_{n}{ }^{\prime} a_{n}(h \mid 02)\left[n j_{n-1}(z)-(n+1) j_{n+1}(z)\right] / \\
m=1
\end{array} \\
& \begin{array}{l}
\sum_{n}^{\prime} a_{n}(h \mid 12)\left\{j_{n+1}(z)+(2 n+3)^{-1}\left[(z / h)^{2}-1\right]\right. \\
\left.m=2 \quad \times\left[(n+1) j_{n}(z)-(n+2) j_{n+2}(z)\right]\right\}=0 . \\
\sum_{n}{ }^{\prime} a_{n}(h \mid 22)\left\{j_{n+2}(z)+\frac{1}{2} z(2 n+5)^{-1}\left[(z / h)^{2}-1\right]\right. \\
\left.\quad \times\left[(n+2) j_{n+1}(z)-(n+3) j_{n+3}(z)\right]\right\}=0 .
\end{array}
\end{aligned}
$$

\section{APPENDIX B}

Several of the longer formulas obtained in the calculation of the quadrupole operator and the absorption cross section are given here.

The constants $C_{\mu}$ in Eq. (66) are given by

$$
\begin{aligned}
C_{0} & =C\left|F_{0}\right|^{2}\left[1+0.406 a_{0}+0.206 a_{0}^{2}\right], \\
C_{ \pm 1} & =C\left|F_{ \pm 1}\right|{ }^{2}\left[1+0.203\left(a_{0} \mp 6^{1 / 2} a_{2}\right)+0.073 a_{0}^{2}\right] \\
C_{ \pm 2} & =C\left|F_{ \pm 2}\right|{ }^{2}\left[1-0.406 a_{0}+0.028 a_{0}^{2}\right]
\end{aligned}
$$

$$
\begin{array}{r}
\alpha=0, \beta=0 \\
\mathrm{~s}=s \hat{z} \\
u=\hat{x} \\
\alpha=\frac{1}{2} \pi, \beta=0 \\
\mathrm{~s}=s \hat{z} \\
u=\hat{y}
\end{array}
$$$$
M=\left(\begin{array}{lll}
0 & 0 & 0 \\
0 & 0 & 0 \\
1 & 0 & 0
\end{array}\right)
$$

with

$$
C=0.174 \kappa \rho_{p}^{2}(0) R_{0}^{3} / \rho_{0} \text {. }
$$

The expressions for the intrinsic quadrupole operators are

$$
\begin{aligned}
Q_{0}= & Q_{0}{ }^{\prime}+0.310\left(\rho_{0} R_{0}{ }^{5} / \kappa\right)^{1 / 2}\left\{k_{20}{ }^{-1}\left(\hbar \omega_{0}\right)^{1 / 2}\right. \\
& \times\left[1+0.355 a_{0}+0.963 a_{0}^{2}\right]\left(q_{0}^{\dagger}+q_{0}\right) \\
& \left.-1.42 k_{22}{ }^{-1}\left(\hbar \omega_{2}\right)^{1 / 2} a_{2}\left(q_{2}^{\dagger}+q_{-2}\right)\right\} \\
Q_{ \pm 1}= & 0.310\left(\rho_{0} R_{0}{ }^{5} / 2 \kappa\right)^{1 / 2}\left\{k_{21}{ }^{-1}\left(\hbar \omega_{1}\right)^{1 / 2}\right. \\
& \left.\times\left[1+0.177\left(a_{0}-6\right)^{1 / 2} a_{2}\right)+0.329 a_{0}{ }^{2}\right]\left(q_{1}^{\dagger}-q_{-1}\right) \\
& \mp k_{2-1}^{-1}\left(\hbar \omega_{-1}\right)^{1 / 2}\left[1+0.177\left(a_{0}+6^{1 / 2} a_{2}\right)\right. \\
& \left.\left.+0.329 a_{0}^{2}\right]\left(q_{-1}^{\dagger}-q_{1}\right)\right\} \\
Q_{ \pm 2}= & Q_{ \pm 2}{ }^{\prime}+0.310\left(\rho_{0} R_{0}{ }^{5} / 2 \kappa\right)^{1 / 2}\left\{k_{22}{ }^{-1}\left(\hbar \omega_{2}\right)^{1 / 2}\right. \\
& \times\left[1-0.355 a_{0}+0.156 a_{0}^{2}\right]\left[\left(q_{2}^{\dagger}+q_{-2}\right)\right. \\
& \left.\left.\mp\left(q_{-2}^{\dagger}+q_{2}\right)\right]-1.42 k_{20}{ }^{-1}\left(\hbar \omega_{0}\right)^{1 / 2} a_{0}\left(q_{0}^{\dagger}+q_{0}\right)\right\} .
\end{aligned}
$$

The $Q_{i j}$ as defined by Eq. (77) are $0.492 e\left(\rho_{0} R_{0}^{5} / \kappa\right)^{1 / 2}$ $\times N_{i j}$ [see Eq. (89)], where

$$
\begin{aligned}
& N_{11}=-k_{20}^{-1}\left(\hbar \omega_{0}\right)^{1 / 2}\left[1+0.355 a_{0}+2.45 a_{2}+0.963 a_{0}^{2}\right] \\
& \times\left(q_{0}^{\dagger}+q_{0}\right)+k_{22}{ }^{-1}\left(3 \hbar \omega_{2}\right)^{1 / 2}\left[1-0.355 a_{0}\right. \\
& \left.+0.816 a_{2}+0.156 a_{0}^{2}\right]\left(q_{2}^{\dagger}+q_{-2}\right) \text {, } \\
& N_{22}=k_{20}{ }^{-1}\left(\hbar \omega_{0}\right)^{1 / 2}\left[1+0.355 a_{0}-2.45 a_{2}+0.963 a_{0}^{2}\right] \\
& \times\left(q_{0}^{\dagger}+q_{0}\right)-k_{22}{ }^{-1}\left(3 \hbar \omega_{2}\right)^{1 / 2}\left[1-0.355 a_{0}\right. \\
& \left.-0.816 a_{2}+0.156 a_{0}^{2}\right]\left(q_{2}^{\dagger}+q_{-2}\right) \text {, } \\
& N_{33}=2 k_{20}{ }^{-1}\left(\hbar \omega_{0}\right)^{1 / 2}\left[1+0.355 a_{0}+0.963 a_{0}^{2}\right] \\
& \times\left(q_{0}^{\dagger}+q_{0}\right)-2 k_{22}{ }^{-1}\left(2 \hbar \omega_{2}\right)^{1 / 2} a_{2}\left(q_{2}^{\dagger}+q_{-2}\right), \\
& N_{12}=N_{21}=i k_{22}{ }^{-1}\left(3 \hbar \omega_{2}\right)^{1 / 2}\left[1-0.355 a_{0}+0.156 a_{0}^{2}\right] \\
& \times\left(q_{-2}^{\dagger}+q_{2}\right) \text {, } \\
& N_{13}=N_{31}=i k_{2-1}^{-1}\left(3 \hbar \omega_{-1}\right)^{1 / 2}\left[1+0.177\left(a_{0}+6^{1 / 2} a_{2}\right)\right. \\
& \left.+0.329 a_{0}^{2}\right]\left(q_{-1}^{\dagger}+q_{1}\right) \\
& N_{23}=N_{32}=-i k_{21}{ }^{-1}\left(3 \hbar \omega_{1}\right)^{1 / 2}\left[1+0.177\left(a_{0}-6^{1 / 2} a_{2}\right)\right. \\
& \left.+0.329 a_{0}^{2}\right]\left(q_{1}^{\dagger}-q_{-1}\right) \text {. }
\end{aligned}
$$

We now list the oscillator strengths for special orientations of the nuclei. These orientations are those of Table I and correspond to those for which each mode has its maximum excitation. The values given are for ${ }_{68} \mathrm{Er}^{166}$. 
$\alpha=0, \beta=\frac{1}{4} \pi$

$$
\begin{aligned}
& \mathbf{s}=\frac{s}{\sqrt{2}}(\hat{x}+\hat{z}) \\
& \mathbf{u}=\frac{1}{\sqrt{2}}(\hat{x}-\hat{z})
\end{aligned}
$$

$\alpha=\frac{1}{4} \pi, \beta=\frac{1}{4} \pi$

$$
\begin{aligned}
& \mathbf{s}=\frac{1}{2} s(\hat{x}+\hat{y}+\sqrt{2} \hat{z}) \\
& \mathbf{u}=\frac{1}{2}(\hat{x}+\hat{y}-\sqrt{2} \hat{z})
\end{aligned}
$$

PHYSICAL REVIEW

$$
M=\frac{1}{2}\left(\begin{array}{rrr}
1 & 0 & -1 \\
0 & 0 & 0 \\
1 & 0 & -1
\end{array}\right)
$$$$
M=\left(\begin{array}{ccc}
1 & 1 & -\sqrt{2} \\
1 & 1 & -\sqrt{2} \\
\sqrt{2} & \sqrt{2} & -2
\end{array}\right)
$$

$$
\begin{aligned}
& f_{20^{10}}=\frac{3}{4}(0.78)\left(N Z / A^{2}\right) R_{0}{ }^{2} \\
& f_{22^{10}}=\frac{1}{4}(0.78)\left(N Z / A^{2}\right) R_{0}{ }^{2} \\
& f_{2 \mu}{ }^{10}=0, \quad \mu \neq 0,2
\end{aligned}
$$

$$
f_{20}{ }^{10}=\frac{3}{4}(0.78)\left(N Z / A^{2}\right) R_{0}{ }^{2}
$$$$
f_{2-2}{ }^{10}=\frac{1}{4}(0.78)\left(N Z / A^{2}\right) R_{0}{ }^{2}
$$$$
f_{2 \mu}{ }^{10}=0, \quad \mu \neq 0, \quad-2 \text {. }
$$

\title{
Convenient Analytic Form for the Deuteron Wave Function*
}

\author{
IAN J. McGeE \\ Department of Physics, University of Wisconsin, Madison, Wisconsin
}

(Received 6 June 1966)

\begin{abstract}
A convenient analytical form for the deuteron wave function is presented which reproduces the static properties of the deuteron, and is in accord with numerical wave functions which fit neutron-proton scattering data up to $300 \mathrm{MeV}$.
\end{abstract}

$\mathrm{D}$ EUTERON wave functions in numerical form have been obtained by several groups ${ }^{1}$ from nucleonnucleon potentials which reproduce the phenomenological phase parameter fits to the two-nucleon scattering data up to several hundred $\mathrm{MeV}$. The object of this note is to present approximate expressions for one of these numerical wave functions which is convenient and accurate for most cases of interest.

The motivation for the analytic form used in this paper comes from several sources. However, it is sufficient for purposes of illustration to indicate the approach from the work of Bertocchi et al..$^{2}$ Their work shows that for neutron-proton potentials describable as a sum of Yukawa forms, the $S$ and $D$ states of the deuteron wave function can be written in coordinate space in the form:

$$
\begin{aligned}
& u(r)=N \int_{0}^{\infty} \sigma_{s}(z) e^{-z r} d z, \\
& w(r)=\rho N \int_{0}^{\infty} \sigma_{d}(z) e^{-z r}\left(1+\frac{3}{z r}+\frac{3}{(z r)^{2}}\right) d z ;
\end{aligned}
$$

* Work supported in part by the University of Wisconsin Research Committee with funds granted by the Wisconsin Alumni Research Foundation and in part by the U. S. Atomic Energy Commission under Contract No. AT(11-1)-881, No. COO-881-69.

${ }_{1}^{1}$ T. Hamada and I. D. Johnston, Nucl. Phys. 34, 382 (1962); N. K. Glendenning and G. Kramer, Phys. Rev. 126, 2159 (1962); S. Gartenhaus, ibid 100, 900 (1956) ; K. E. Lassila, M. H. Hull, Jr., H. M. Ruppel, F. A. McDonald, and G. Breit, ibid. 126, 881 (1962); H. Feshbach and E. L. Lomon, Ann. Phys. (N.Y.) 29, 19 (1964)

${ }^{2}$ L. Bertocchi, C. Ceolin, and M. Tonin, Nuovo Cimento 18 770 (1960). Although these authors only considered the $S$-state wave function, the formalism is easily extended to include all angular momentum wave functions. The author would like to and in momentum space as

$$
\begin{gathered}
\tilde{u}(p)=N \int_{0}^{\infty} \frac{\sigma_{s}(z) d z}{p^{2}+z^{2}}=\frac{N}{p^{2}+\alpha^{2}}+N \int_{\alpha+\lambda}^{\infty} \frac{\hat{\sigma}_{s}(z) d z}{p^{2}+z^{2}} \\
-\tilde{w}(p)=\rho N \int_{0}^{\infty} \frac{\sigma_{d}(z) d z}{p^{2}+z^{2}}=\frac{\rho N}{p^{2}+\alpha^{2}}+\rho N \int_{\alpha+\lambda}^{\infty} \frac{\hat{\sigma}_{d}(z) d z}{p^{2}+z^{2}},
\end{gathered}
$$

where

$$
\sigma_{i}(z)=\delta(z-\alpha)+\hat{\sigma}_{i}(z) \theta(z-\alpha-\lambda), \quad i=s, d,
$$

the function $\theta$ being the unit step function. Here $\lambda$ is the minimum decay constant which appears in the potential, $\alpha$ is given by the deuteron binding energy $\epsilon$, $\alpha=(m \epsilon)^{1 / 2}, \rho$ is the asymptotic $D$ to $S$ ratio, and $N$ is the wave-function normalization, given in terms of the deuteron effective range $\rho(-\epsilon,-\epsilon)$ by

$$
N^{2}=2 \alpha /[1-\alpha \rho(-\epsilon,-\epsilon)] \text {. }
$$

The weight functions $\sigma_{i}(z)$ in Eqs. (1) and (2) are subject to the subsidiary conditions ${ }^{3}$

$$
\begin{aligned}
& \int_{0}^{\infty} \sigma_{s}(z) d z=0, \\
& \int_{0}^{\infty} \sigma_{d}(z) z^{m} d z=0, \quad m=-2,0,2 .
\end{aligned}
$$

These sum rules are required to guarantee that the wave functions be finite at the origin and have the correct indicial behavior.

thank Professor L. Durand, III, for calling his attention to this. See also Ref. 3. 